Bulletin of the Seismological Society of America. Vol. 48, pp. 133-146. April 1958

\title{
MECHANISMS OF THE AFTERSHOCKS OF THE KERN COUNTY, CALIFORNIA, EARTHQUAKE OF 1952
}

\author{
By Markus Båth and Charles F. Richter
}

\begin{abstract}
Using directions of first motion of longitudinal waves recorded at near-by stations, the orientation of fault traces and the nature of fault motions have been deduced for fifty-seven earthquakes of the Kern County aftershock series. Unlike the main shock, the aftershocks exhibit considerable strike slip with left-hand strike slip dominating on and to the south of the White Wolf fault and right-hand strike slip and dip slip to the north of it. The two last-mentioned mechanisms represent a secondary strain release, beginning not earlier than thirty-seven hours after the main shock.
\end{abstract}

\section{INTRODUCTION}

EARLIER studies of faulting mechanism in southern California by means of seismograms have been made by Gutenberg (1941), using first motions of longitudinal waves, and by Dehlinger (1952), using shear-wave vibrational directions. For the main Kern County earthquake on July 21, 1952, a solution was given by Gutenberg (1955b) by means of longitudinal and transverse waves at near and distant stations. The result was a fault plane dipping $60^{\circ}$ to $66^{\circ}$ toward $\mathrm{E} 50^{\circ} \mathrm{S}$ and a slip directed upward and northeastward in the southeastern block relative to the northwestern block. The dip-slip component was about 1.4 times the strike-slip component. In the present paper we shall investigate the mechanism for a number of the Kern County aftershocks as a contribution to an understanding of the mechanics of the whole system.

\section{Observational Data}

The observations of the direction of initial motion of the $\mathrm{P}$ waves are given in table 1 for fifty-seven aftershocks selected from Richter's (1955) list, including all of magnitudes $\geqq 5.0$ and a number of smaller shocks of special interest.

The following notation is used:

No. = shock number, assigned by Richter (1955)

$\mathrm{M}$ = magnitude

$\mathrm{C}=$ compression

$\mathrm{D}=$ dilatation

$\mathrm{dC}=$ small dilatation followed by large compression

$\mathrm{cD}=$ small compression followed by large dilatation

$(\quad)=$ questionable reading; if no parenthesis is given, the reading is very clear

- = beginning gradual with indefinite direction, or obscured

Blank entry means that no record exists or that it has not been available for inspection.

The following abbreviations are used for the stations (see Gutenberg, 1955a, concerning location and instrumentation):

$\mathrm{P}=$ Pasadena, $\mathrm{MW}=$ Mount Wilson, $\mathrm{R}=$ Riverside, $\mathrm{Pr}=$ Palomar, $\mathrm{LJ}=\mathrm{La}$ Jolla, $\mathrm{SB}=$ Santa Barbara, CL $=$ China Lake, $\mathrm{H}=$ Haiwee, $\mathrm{T}=$ Tinemaha, $\mathrm{BB}=$ Big Bear, $\mathrm{Bt}=$ Barrett, $\mathrm{Ch}=$ Chuchupate, $\mathrm{By}=$ Berkeley, $\mathrm{MH}=$ Mount Hamilton, $\mathrm{PA}=$ Palo Alto, $\mathrm{F}=$ Fresno, $\mathrm{M}=$ Mineral, $\mathrm{BC}=$ Boulder City, $\mathrm{Ro}=$ Reno.

Manuscript received for publication March 18, 1957. 
TABLE 1

Observational Data

\begin{tabular}{|c|c|c|c|c|c|c|c|c|c|c|c|c|}
\hline No. & $\begin{array}{c}\text { Date. } \\
\text { time } \\
\text { (G.C.T.) }\end{array}$ & $\begin{array}{l}\text { Lat., } \\
\text { long. }\end{array}$ & M & $\mathrm{P}$ & MW & $\mathrm{R}$ & $\mathrm{Pr}$ & $\mathrm{LJ}$ & $\mathrm{sB}$ & $\mathrm{CL}$ & $\mathrm{H}$ & $\mathrm{T}$ \\
\hline & 1952 & deg. min. & & & & & & & & & & \\
\hline \multirow[t]{2}{*}{40} & July 21 & 3514 & 5.1 & (C) & - & - & - & - & - & $(\mathrm{cD})$ & $\mathrm{D}$ & $\mathrm{D}$ \\
\hline & $\begin{array}{l}17: 43 \\
\text { July } 21\end{array}$ & $\begin{array}{rl}118 & 32 \\
35 & 08\end{array}$ & 5.5 & & & & & 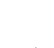 & $C$ & & & \\
\hline 46 & $\begin{array}{l}\text { July } 21 \\
19: 41\end{array}$ & 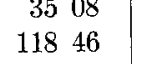 & 0.8 & $\mathrm{C}$ & - & $\mathrm{C}$ & C & - & $\mathrm{C}$ & 1) & D & $\mathrm{D}$ \\
\hline 58 & July 22 & $\begin{array}{lll}35 & 12.5\end{array}$ & 4.4 & $\mathrm{C}$ & - & (D) & - & - & $(\mathrm{dC})$ & (C) & - & (D) \\
\hline \multirow[t]{2}{*}{59} & $\begin{array}{l}03: 21 \\
\text { July } 22\end{array}$ & $\begin{array}{rl}118 & 37.5 \\
34 & 52\end{array}$ & 4.1 & $\mathrm{D}$ & - & $\mathrm{C}$ & $\mathrm{C}$ & - & $\mathrm{cD}$ & (D) & - & $\mathrm{dC}$ \\
\hline & $07: 45$ & 11852 & & & & & & & & & & \\
\hline \multirow[t]{2}{*}{60} & July 22 & 3505 & 4.4 & $\mathrm{C}$ & C & (C) & $\mathrm{cD}$ & - & D & $\mathrm{D}$ & (c) $\mathrm{D}$ & - \\
\hline & 08:16 & $\begin{array}{r}11835 \\
3500\end{array}$ & 41 & & & & & & & & & \\
\hline 61 & $\begin{array}{l}\text { July } 22 \\
08: 21\end{array}$ & $\begin{array}{r}3500 \\
11900\end{array}$ & 4.1 & C & (c) D & - & $\mathrm{C}$ & - & (D) & $\mathrm{C}$ & - & $\mathrm{cD}$ \\
\hline \multirow[t]{2}{*}{62} & July 22 & 3505 & 4.7 & $\mathrm{D}$ & (D) & $\mathrm{D}$ & $\mathrm{dC}$ & - & $\mathrm{C}$ & $\mathrm{C}$ & (C) & $\mathrm{D}$ \\
\hline & 08:48 & 11845 & & & & & & & & & & \\
\hline \multirow[t]{2}{*}{63} & July 22 & $\begin{array}{r}3514 \\
118 \quad 36\end{array}$ & 4.5 & D & $\mathrm{D}$ & - & $\mathrm{dC}$ & - & $\mathrm{dC}$ & $\mathrm{C}$ & $\mathrm{C}$ & D \\
\hline & $09: 10$ & $\begin{array}{r}118 \quad 36 \\
3502\end{array}$ & 4.1 & $\mathrm{dC}$ & - & $\ldots$ & $\mathrm{D}$ & - & $\mathrm{D}$ & (C) & D & $\mathrm{cD}$ \\
\hline 64 & $10: 20$ & 11900 & & ac & - & & CD & & $D$ & & & \\
\hline \multirow[t]{2}{*}{65} & July 22 & $\begin{array}{ll}35 & 03\end{array}$ & 3.8 & $\mathrm{D}$ & D & - & - & - & - & - & - & - \\
\hline & $10: 44$ & 11830 & & & & & & & & & & \\
\hline \multirow[t]{2}{*}{66} & July 22 & 3500 & 4.8 & D & D & (C) & $\mathrm{dC}$ & - & $\mathrm{dC}$ & $\mathrm{C}$ & (C) & $\mathrm{D}$ \\
\hline & $13: 32$ & 11900 & & & & & & & & & & \\
\hline 68 & July 22 & $34 \quad 54$ & 4.3 & D & (D) & - & $(\mathrm{dC})$ & - & $\mathrm{C}$ & - & - & $\mathrm{D}$ \\
\hline 69 & $\begin{array}{l}\text { July } 22 \\
15: 03\end{array}$ & $\begin{array}{rr}30 & 14 \\
118 & 32\end{array}$ & & & D & - & & & & & & D \\
\hline \multirow[t]{2}{*}{70} & July 22 & 3500 & 4.1 & $\mathrm{D}$ & - & - & C & - & $\mathrm{C}$ & $\mathrm{C}$ & - & (c) D \\
\hline & $17: 53$ & 11900 & & & & & & 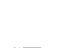 & & $C$ & & \\
\hline 71 & $\begin{array}{l}\text { July } 22 \\
\text { 19:09 }\end{array}$ & $\begin{array}{r}35 \quad 13 \\
118 \quad 28\end{array}$ & 4.3 & (C) & $\mathrm{C}$ & - & - & - & - & $\mathrm{C}$ & $\mathrm{D}$ & $\mathrm{D}$ \\
\hline \multirow[t]{2}{*}{73} & July 22 & 3504 & 4.2 & $\mathrm{D}$ & $\mathrm{D}$ & - & - & - & - & $\mathrm{D}$ & - & - \\
\hline & $21: 02$ & 11846 & & & & & & & & & & \\
\hline 74 & $\begin{array}{l}\text { July } 22 \\
22: 32\end{array}$ & 3501.5 & 4.7 & C & $\mathrm{C}$ & - & $\mathrm{C}$ & - & $\mathrm{dC}$ & $\mathrm{C}$ & (C) & $\mathrm{C}$ \\
\hline \multirow[t]{2}{*}{75} & $\begin{array}{l}22: 32 \\
\text { July } 23\end{array}$ & $\begin{array}{c}11855.5 \\
35\end{array}$ & 6.1 & $\mathrm{C}$ & - & - & - & - & $\mathrm{C}$ & $\mathrm{C}$ & (C) & D \\
\hline & $00: 39$ & 11835 & & & & & & & & 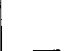 & & \\
\hline 76 & $\begin{array}{l}\text { July } 23 \\
00: 43\end{array}$ & $\begin{array}{r}35.0 \\
1190\end{array}$ & 4.4 & - & - & - & - & - & $\mathrm{C}$ & 一 & - & - \\
\hline 77 & $\begin{array}{l}\text { July } 23 \\
00: 48\end{array}$ & $\begin{array}{r}119.0 \\
35 \quad 22 \\
118 \quad 35\end{array}$ & 4.6 & $\mathrm{D}$ & $\mathrm{D}$ & - & (C) & - & $\mathrm{C}$ & $\mathrm{C}$ & $\mathrm{dC}$ & C \\
\hline
\end{tabular}


TABLE 1-Continued

\begin{tabular}{|c|c|c|c|c|c|c|c|c|c|c|c|}
\hline $\mathrm{BB}$ & $\mathrm{Bt}$ & $\mathrm{Ch}$ & By & $\mathrm{MH}$ & PA & F & M & $\mathrm{BC}$ & Ro & Additional data & No. \\
\hline- & - & & $\mathrm{cD}$ & - & D & (D) & & $\mathrm{C}$ & & & 40 \\
\hline (C) & $\mathrm{C}$ & & D & D & $\mathrm{dC}$ & $\mathrm{D}$ & & $\mathrm{C}$ & & & 46 \\
\hline- & (D) & & (C) & - & 一 & $\mathrm{D}$ & - & - & - & & 58 \\
\hline- & $(\mathrm{cD})$ & & - & (C) & - & $\mathrm{D}$ & (D) & - & - & White Oak: D & 59 \\
\hline $\mathrm{cD}$ & (C) & & - & D & -. & $\mathrm{D}$ & - & - & - & & 60 \\
\hline (c) D & - & & - & - & - & D & - & - & (C) & White Oak: D & 61 \\
\hline- & - & & - & (C) & - & $\mathrm{D}$ & - & - & - & & 62 \\
\hline $\mathrm{eD}$ & (C) & & D & $\mathrm{eD}$ & - & D & - & $\mathrm{cD}$ & - & . & 63 \\
\hline- & $\mathrm{C}$ & & 一 & - & - & $\mathrm{D}$ & (D) & - & - & & 64 \\
\hline $\mathrm{cD}$ & $(\mathrm{eD})$ & & - & - & - & $\mathrm{D}$ & & - & - & & 65 \\
\hline $\mathrm{C}$ & - & & (D) & $\mathrm{D}$ & (C) & $\mathrm{D}$ & & - & $\mathrm{D}$ & & 66 \\
\hline - & - & & - & - & - & $\mathrm{D}$ & & - & - & & 68 \\
\hline- & - & & (D) & (D) & - & (D) & & - & - & & 69 \\
\hline - & - & & - & - & - & - & & - & - & & 70 \\
\hline (cD) & - & & (D) & (c) $\mathrm{D}$ & - & - & & $\mathrm{C}$ & - & & 71 \\
\hline - & - & & $\mathrm{D}$ & (D) & - & $\mathrm{C}$ & - & - & - & & 73 \\
\hline $\mathrm{C}$ & - & & 一 & D & - & $\mathrm{C}$ & - & - & - & & 74 \\
\hline- & - & & D & D & $\mathrm{D}$ & D & 一 & $\mathrm{C}$ & $\mathrm{D}$ & Santa Clara: D & 75 \\
\hline- & - & & - & - & - & - & - & - & - & & 76 \\
\hline - & - & & - & C & C & - & - & (C) & - & & 77 \\
\hline
\end{tabular}


TABLE 1-Continued

\begin{tabular}{|c|c|c|c|c|c|c|c|c|c|c|c|c|}
\hline No. & $\begin{array}{c}\text { Date. } \\
\text { time } \\
\text { (G.C.T.) }\end{array}$ & $\begin{array}{l}\text { Lat., } \\
\text { long. }\end{array}$ & M & $P$ & MW & $\mathrm{R}$ & $\operatorname{Pr}$ & LJ & $\mathrm{SB}$ & $\mathrm{CL}$ & $\mathrm{H}$ & $T$ \\
\hline & 1952 & deg. min. & & & & & & & & & & \\
\hline 78 & $\begin{array}{l}\text { July } 23 \\
03: 19\end{array}$ & $\begin{array}{r}35 \quad 22 \\
118 \quad 35\end{array}$ & 5.0 & $\mathrm{D}$ & D & (D) & (D) & - & $\mathrm{C}$ & $\mathrm{C}$ & (d) $\mathrm{C}$ & $\mathrm{C}$ \\
\hline 79 & $\begin{array}{l}\text { July } 23 \\
03: 49\end{array}$ & $\begin{array}{r}3517 \\
118 \quad 33\end{array}$ & 4.7 & $D$ & (D) & (D) & $\mathrm{C}$ & - & - & $\mathrm{C}$ & $\mathrm{dC}$ & $\mathrm{C}$ \\
\hline 80 & $\begin{array}{l}\text { July } 23 \\
04: 02\end{array}$ & $\begin{array}{r}3522 \\
11835\end{array}$ & 4.7 & $\mathrm{D}$ & $\mathrm{D}$ & (D) & D & - & $\mathrm{C}$ & $\mathrm{C}$ & $\mathrm{dC}$ & $\mathrm{C}$ \\
\hline 81 & $\begin{array}{l}\text { July } 23 \\
05: 46\end{array}$ & $\begin{array}{r}35 \quad 23 \\
118 \quad 34\end{array}$ & 4.7 & (D) & $\mathrm{D}$ & - & $\mathrm{cD}$ & - & - & $\mathrm{C}$ & $\mathrm{C}$ & $\mathrm{C}$ \\
\hline 82 & $\begin{array}{l}\text { July } 23 \\
06: 11\end{array}$ & $\begin{array}{r}3516 \\
11827\end{array}$ & 4.2 & $\mathrm{D}$ & (D) & - & - & -- & C & $\mathrm{C}$ & - & $\mathrm{D}$ \\
\hline 83 & $\begin{array}{l}\text { July } 23 \\
06: 26\end{array}$ & $\begin{array}{r}3522 \\
118 \quad 35\end{array}$ & 4.0 & - & - & - & - & - & C & $\mathrm{C}$ & C & C \\
\hline 84 & $\begin{array}{l}\text { July } 23 \\
06: 54\end{array}$ & $\begin{array}{r}3522 \\
11835\end{array}$ & 4.2 & $\mathrm{D}$ & - & - & - & - & $\mathrm{D}$ & (C) & (C) & $\mathrm{C}$ \\
\hline 85 & $\begin{array}{l}\text { July } 23 \\
07: 37\end{array}$ & $\begin{array}{r}3517 \\
11833\end{array}$ & 4.8 & $\mathrm{D}$ & $\mathrm{D}$ & - & D & - & $\mathrm{dC}$ & $\mathrm{C}$ & $\mathrm{dC}$ & $\mathrm{dC}$ \\
\hline 86 & $\begin{array}{l}\text { July } 23 \\
07: 53\end{array}$ & $\begin{array}{r}3500 \\
11850\end{array}$ & 5.4 & D & $\mathrm{D}$ & $\mathrm{cD}$ & $\mathrm{C}$ & - & $\mathrm{cD}$ & $\mathrm{C}$ & (D) & $\mathrm{D}$ \\
\hline 87 & $\begin{array}{l}\text { July } 23 \\
09: 39\end{array}$ & $\begin{array}{r}3515 \\
11829\end{array}$ & 4.2 & $\mathrm{D}$ & $\mathrm{D}$ & (D) & - & - & $\mathrm{C}$ & $\mathrm{C}$ & $\mathrm{D}$ & $(\mathrm{dC})$ \\
\hline 88 & $\begin{array}{l}\text { July } 23 \\
10: 54\end{array}$ & $\begin{array}{r}3519 \\
11830\end{array}$ & 4.1 & D & D & - & $\mathrm{eD}$ & - & (C) & $\mathrm{C}$ & $\mathrm{D}$ & $\mathrm{D}$ \\
\hline 89 & $\begin{array}{l}\text { July } 23 \\
13: 17\end{array}$ & $\begin{array}{r}3513 \\
11849\end{array}$ & 5.7 & $\mathrm{C}$ & (c) D & C & $\mathrm{C}$ & - & C & D & $\mathrm{D}$ & $\mathrm{D}$ \\
\hline 97 & $\begin{array}{l}\text { July } 23 \\
18: 14\end{array}$ & $\begin{array}{r}3500 \\
11850\end{array}$ & 5.2 & $\mathrm{C}$ & $\mathrm{D}$ & D & D & - & $\mathrm{dC}$ & $\mathrm{C}$ & $\mathrm{dC}$ & $d C$ \\
\hline 113 & $\begin{array}{l}\text { July } 25 \\
07: 04\end{array}$ & $\begin{array}{r}3524 \\
11835\end{array}$ & 4.1 & $\mathrm{C}$ & 一 & (C) & - & - & - & C & C & $\mathrm{D}$ \\
\hline 115 & $\begin{array}{l}\text { July } 25 \\
13: 13\end{array}$ & $\begin{array}{r}3519 \\
11830\end{array}$ & 5.0 & D & $\mathrm{D}$ & $\mathrm{cD}$ & cD & - & - & $D$ & $\mathrm{D}$ & $\mathrm{D}$ \\
\hline 116 & $\begin{array}{l}\text { July } 25 \\
14: 35\end{array}$ & $\begin{array}{r}3508 \\
11846\end{array}$ & 4.4 & D & (cD) & $\mathrm{D}$ & (D) & - & (C) & $\mathrm{C}$ & - & $\mathrm{D}$ \\
\hline 117 & $\begin{array}{l}\text { July } 25 \\
19: 10\end{array}$ & $\begin{array}{r}3519 \\
11830\end{array}$ & 5.7 & $\mathrm{D}$ & $\mathrm{D}$ & $\mathrm{D}$ & (D) & - & $\mathrm{dC}$ & C & $D$ & $\mathrm{D}$ \\
\hline 118 & $\begin{array}{l}\text { July } 25 \\
19: 43\end{array}$ & $\begin{array}{r}3519 \\
118 \quad 30\end{array}$ & 5.7 & $\mathrm{D}$ & - & C & $\mathrm{C}$ & - & $\mathrm{C}$ & C & $\mathrm{D}$ & $\mathrm{D}$ \\
\hline 119 & $\begin{array}{l}\text { July } 25 \\
20: 06\end{array}$ & $\begin{array}{r}3519 \\
118 \quad 30\end{array}$ & 4.8 & - & - & - & - & - & - & - & $\mathrm{D}$ & $\mathrm{D}$ \\
\hline 120 & $\begin{array}{l}\text { July } 26 \\
01: 02\end{array}$ & $\begin{array}{r}3519 \\
11830\end{array}$ & 4.2 & $\mathrm{D}$ & (D) & $\mathrm{C}$ & $\mathrm{C}$ & 一 & $\mathrm{D}$ & $\mathrm{D}$ & $\mathrm{cD}$ & $\mathrm{D}$ \\
\hline
\end{tabular}


TABLE 1-Continued

\begin{tabular}{|c|c|c|c|c|c|c|c|c|c|c|c|}
\hline BB & $\mathrm{Bt}$ & $\mathrm{Ch}$ & By & MF & $\mathrm{PA}$ & $F$ & M & $\mathrm{BC}$ & Ro & Additional data & No. \\
\hline $\mathrm{D}$ & $\mathrm{D}$ & & $\mathrm{C}$ & $\mathrm{C}$ & (C) & $\mathrm{C}$ & - & $\mathrm{C}$ & $\mathrm{D}$ & & 78 \\
\hline- & & & $(\mathrm{dC})$ & (C) & - & $\mathrm{D}$ & - & $\mathrm{C}$ & - & & 79 \\
\hline $\mathrm{D}$ & - & & - & $\mathrm{C}$ & - & $\mathrm{D}$ & - & $\mathrm{C}$ & - & & 80 \\
\hline$(\mathrm{dC})$ & (D) & & (C) & - & - & 一 & - & - & - & & 81 \\
\hline $\mathrm{C}$ & $\mathrm{D}$ & $\mathrm{C}$ & $\mathrm{D}$ & $\mathrm{C}$ & - & - & - & $\mathrm{C}$ & - & & 82 \\
\hline- & - & & - & $\mathrm{C}$ & - & - & - & (C) & - & & 83 \\
\hline- & - & & - & - & - & - & - & $\mathrm{C}$ & - & & 84 \\
\hline $\mathrm{dC}$ & $\mathrm{D}$ & & - & $\mathrm{D}$ & - & $\mathrm{D}$ & - & $\mathrm{C}$ & - & & 85 \\
\hline- & $\mathrm{C}$ & & $\mathrm{cD}$ & $\mathrm{C}$ & - & $\mathrm{D}$ & - & $\mathrm{C}$ & - & & 86 \\
\hline $\mathrm{eD}$ & - & & - & $\mathrm{D}$ & - & $\mathrm{D}$ & - & (C) & - & & 87 \\
\hline - & $\mathrm{cD}$ & & $\mathrm{C}$ & - & - & - & - & $\mathrm{C}$ & (D) & & 88 \\
\hline $\mathrm{C}$ & $\mathrm{C}$ & & - & $\mathrm{C}$ & - & $\mathrm{D}$ & $\mathrm{D}$ & (D) & $\mathrm{D}$ & & 89 \\
\hline $\mathrm{D}$ & $\mathrm{D}$ & & $\mathrm{D}$ & $\mathrm{C}$ & $\mathrm{D}$ & $\mathrm{C}$ & - & $\mathrm{C}$ & - & Dalton: (D) & 97 \\
\hline 一 & - & & (c) D & $\mathrm{D}$ & (C) & $\mathrm{D}$ & - & C & - & & 113 \\
\hline (c) $\mathrm{D}$ & (D) & $\mathrm{C}$ & $\mathrm{cD}$ & (cD) & (C) & (D) & (C) & $\mathrm{cD}$ & $\mathrm{D}$ & & 115 \\
\hline- & $\mathrm{D}$ & $\mathrm{C}$ & - & - & - & $\mathrm{D}$ & (D) & (C) & - & & 116 \\
\hline (C) & (D) & $\mathrm{C}$ & $\mathrm{C}$ & - & $\mathrm{C}$ & (D) & 一 & $\mathrm{C}$ & $\mathrm{D}$ & Santa Clara: dC & 117 \\
\hline - & $\mathrm{C}$ & C & C & $\mathrm{C}$ & $\mathrm{C}$ & $\mathrm{C}$ & (C) & $\mathrm{C}$ & $\mathrm{D}$ & & 118 \\
\hline - & - & $\mathrm{D}$ & - & $\mathrm{D}$ & (D) & $\mathrm{D}$ & $\mathrm{D}$ & $\mathrm{C}$ & - & & 119 \\
\hline $\mathrm{C}$ & $(\mathrm{cD})$ & & - & (D) & - & - & - & - & - & & 120 \\
\hline
\end{tabular}


TABLE 1-Continued

\begin{tabular}{|c|c|c|c|c|c|c|c|c|c|c|c|c|}
\hline No. & $\begin{array}{c}\text { Date, } \\
\text { time } \\
\text { (G.C.T.) }\end{array}$ & $\begin{array}{l}\text { Lat., } \\
\text { long. }\end{array}$ & M & $\mathrm{P}$ & $M W$ & $R$ & $\operatorname{Pr}$ & LJ & SB & CL & $\mathrm{H}$ & $\mathrm{T}$ \\
\hline & 1952 & deg. $\min$ & & & & & & & & & & \\
\hline 140 & $\begin{array}{l}\text { July } 29 \\
05: 56\end{array}$ & $\begin{array}{r}3523 \\
11851\end{array}$ & 3.9 & $\mathrm{C}$ & $\mathrm{C}$ & $\mathrm{C}$ & (C) & - & $(\mathrm{d}) \mathrm{C}$ & (c) D & - & $\mathrm{C}$ \\
\hline 141 & July 29 & 3523 & 6.1 & $\mathrm{C}$ & $\mathrm{C}$ & $\mathrm{C}$ & $\mathrm{C}$ & $\mathrm{C}$ & $\mathrm{C}$ & $\mathrm{D}$ & $\mathrm{C}$ & $\mathrm{C}$ \\
\hline 142 & $\begin{array}{l}\text { July } 29 \\
07: 56\end{array}$ & $\begin{array}{r}3523 \\
11846\end{array}$ & 3.8 & (D) & - & $\mathrm{dC}$ & $(\mathrm{dC})$ & - & - & C & - & $\mathrm{C}$ \\
\hline 143 & $\begin{array}{l}\text { July } 29 \\
08: 02\end{array}$ & $\begin{array}{r}3524 \\
11849\end{array}$ & 5.1 & D & D & $\mathrm{D}$ & $D$ & - & C & $\mathrm{C}$ & $\mathrm{dC}$ & $\mathrm{C}$ \\
\hline 144 & $\begin{array}{l}\text { July } 29 \\
08: 08\end{array}$ & $\begin{array}{r}3526 \\
11847\end{array}$ & 3.8 & $\mathrm{D}$ & $\mathrm{D}$ & $\mathrm{D}$ & $\mathrm{D}$ & - & C & $\mathrm{C}$ & $\mathrm{C}$ & $\mathrm{C}$ \\
\hline 155 & $\begin{array}{l}\text { July } 31 \\
12: 09\end{array}$ & $\begin{array}{rr}35 & 19.5 \\
118 & 36.5\end{array}$ & 5.8 & $\mathrm{D}$ & $\mathrm{D}$ & $\mathrm{D}$ & $\mathrm{D}$ & & - & $\mathrm{C}$ & $\mathrm{cD}$ & $\mathrm{D}$ \\
\hline 161 & $\begin{array}{l}\text { Aug. } 1 \\
13: 05\end{array}$ & $\begin{array}{rr}34 & 54 \\
118 & 57\end{array}$ & 5.1 & D & $\mathrm{D}$ & $\mathrm{C}$ & C & & $\mathrm{dC}$ & $\mathrm{C}$ & $\mathrm{C}$ & (D) \\
\hline 177 & $\begin{array}{l}\text { Aug. } 10 \\
19: 44\end{array}$ & $\begin{array}{r}3500 \\
11900\end{array}$ & 4.1 & $\mathrm{C}$ & - & $\mathrm{C}$ & $\mathrm{C}$ & & $\mathrm{D}$ & $\mathrm{D}$ & $\mathrm{D}$ & $\mathrm{cD}$ \\
\hline 194 & $\begin{array}{l}\text { Aug. } 22 \\
22: 41\end{array}$ & $\begin{array}{r}3520 \\
11855\end{array}$ & 5.8 & $\mathrm{C}$ & $\mathrm{C}$ & $\mathrm{C}$ & $\mathrm{C}$ & & $\mathrm{C}$ & $\mathrm{D}$ & C & $\mathrm{C}$ \\
\hline 216 & $\begin{array}{l}\text { Sept. } 12 \\
10: 35\end{array}$ & $\begin{array}{r}3500 \\
11903\end{array}$ & 4.5 & $\mathrm{C}$ & (C) & $\mathrm{C}$ & $\mathrm{C}$ & & (c) D & $\mathrm{D}$ & D & $\mathrm{eD}$ \\
\hline 241 & $\begin{array}{c}\text { Nov. } 7 \\
08: 56 \\
1953\end{array}$ & $\begin{array}{r}3500 \\
11905\end{array}$ & 4.6 & C & (D) & $\mathrm{C}$ & (C) & & D & $\mathrm{D}$ & D & (c) $D$ \\
\hline 266 & $\begin{array}{l}\text { May } 25 \\
03: 24\end{array}$ & $\begin{array}{r}3500 \\
11901\end{array}$ & 4.8 & $\mathrm{D}$ & & C & & & C & C & D & \\
\hline 275 & $\begin{array}{l}\text { Dec. } 15 \\
12: 45 \\
1954\end{array}$ & $\begin{array}{r}3513 \\
11849\end{array}$ & 4.6 & $\mathrm{C}$ & $\mathrm{C}$ & & $\mathrm{C}$ & & & - & $\mathrm{D}$ & \\
\hline 276 & $\begin{array}{l}\text { Jan. } 12 \\
23: 34\end{array}$ & $\begin{array}{r}3500 \\
11901\end{array}$ & 5.9 & $\mathrm{C}$ & - & C & & & D & $\mathrm{C}$ & D & $\mathrm{D}$ \\
\hline 279 & $\begin{array}{l}\text { Jan. } 27 \\
14: 20\end{array}$ & $\begin{array}{r}3509 \\
11838\end{array}$ & 5.0 & $\mathrm{D}$ & $\mathrm{D}$ & $\mathrm{C}$ & & & $\mathrm{D}$ & $\mathrm{D}$ & D & $\mathrm{D}$ \\
\hline 281 & $\begin{array}{l}\text { Feb. } 7 \\
00: 10\end{array}$ & $\begin{array}{r}3502 \\
11906\end{array}$ & 4.4 & $\mathrm{C}$ & $\mathrm{C}$ & $\mathrm{C}$ & $\mathrm{C}$ & & D & $\mathrm{D}$ & $\mathrm{D}$ & $\mathrm{cD}$ \\
\hline 285 & $\begin{array}{l}\text { May } 23 \\
23: 53\end{array}$ & $\begin{array}{r}3459 \\
11859\end{array}$ & 5.1 & $\mathrm{C}$ & C & C & C & & $\mathrm{dC}$ & $\mathrm{D}$ & $\mathrm{D}$ & - \\
\hline
\end{tabular}


TABLE 1-Continued

\begin{tabular}{|c|c|c|c|c|c|c|c|c|c|c|c|}
\hline $\mathrm{BB}$ & $\mathrm{Bt}$ & $\mathrm{Ch}$ & By & MH & $\mathrm{PA}$ & F & M & $\mathrm{BC}$ & Ro & Additional data & No. \\
\hline $\mathrm{dC}$ & $\mathrm{C}$ & $\mathrm{D}$ & - & - & - & $\mathrm{D}$ & - & 一 & - & & 140 \\
\hline $\mathrm{D}$ & $\mathrm{D}$ & & $\mathrm{D}$ & $\mathrm{D}$ & $\mathrm{D}$ & $\mathrm{D}$ & - & D & $\mathrm{dC}$ & & 141 \\
\hline - & $\mathrm{C}$ & & - & (C) & - & 一 & 一 & - & - & & 142 \\
\hline $\mathrm{D}$ & $\mathrm{D}$ & $\mathrm{D}$ & $\mathrm{D}$ & D & - & $\mathrm{D}$ & - & $\mathrm{C}$ & 一 & & 143 \\
\hline$(\mathrm{dC})$ & $\mathrm{d} C$ & $\mathrm{D}$ & - & $\mathrm{C}$ & 一 & - & - & - & 一 & & 144 \\
\hline $\mathrm{C}$ & D & $\mathrm{C}$ & $\mathrm{D}$ & $\mathrm{D}$ & $\mathbf{D}$ & $\mathrm{D}$ & $\mathrm{D}$ & $\mathrm{C}$ & $\mathrm{D}$ & Dalton: (C), & 155 \\
\hline $\mathrm{C}$ & - & & $\mathrm{D}$ & D & - & D & - & - & & Dalton: (C) & 161 \\
\hline$\longrightarrow$ & $\mathrm{C}$ & & & & & & & & & $\begin{array}{l}\text { Woody: D, } \\
\text { Havilah: } \mathrm{C}\end{array}$ & 177 \\
\hline $\mathrm{C}$ & & D & $\mathrm{D}$ & $\mathrm{D}$ & $\mathrm{C}$ & $\mathrm{cD}$ & $一$ & & $\mathrm{cD}$ & $\begin{array}{l}\text { Dalton: (D), } \\
\text { Woody: C }\end{array}$ & 194 \\
\hline $\mathrm{C}$ & & $\mathrm{C}$ & & & & & & & & & 216 \\
\hline $\mathrm{C}$ & & & & & & & & & & Dalton: (D) & 241 \\
\hline & $\mathrm{C}$ & & & & & & & & & $\begin{array}{l}\text { Woody: D, } \\
\text { Fort Tejon: C } \\
\text { Fort Tejon: D, } \\
\text { King Ranch: C }\end{array}$ & $\begin{array}{l}266 \\
275\end{array}$ \\
\hline $\mathrm{C}$ & $\mathrm{D}$ & & & & & & & & & $\begin{array}{l}\text { King Ranch: C, } \\
\text { Isabella: (C) }\end{array}$ & 276 \\
\hline $\mathrm{C}$ & $\mathrm{C}$ & & & & & & & & & $\begin{array}{l}\text { Woody: D, } \\
\text { Fort Tejon: C, } \\
\text { Isabella: C }\end{array}$ & 279 \\
\hline- & $\mathrm{C}$ & & & & & & & & & $\begin{array}{l}\text { Woody: (D), } \\
\text { Fort Tejon: D, } \\
\text { Dalton: D }\end{array}$ & 281 \\
\hline $\mathrm{C}$ & $\mathrm{C}$ & & & & & & & & & $\begin{array}{l}\text { King Ranch: D, } \\
\text { Isabella: D }\end{array}$ & 285 \\
\hline
\end{tabular}


The following locations were not included in Gutenberg's (1955a) paper:

Isabella, $35^{\circ} 39^{\prime} \mathrm{N}, 118^{\circ} 29^{\prime} \mathrm{W}$; Reno (Nevada), $39^{\circ} 32^{\prime} \mathrm{N}, 119^{\circ} 49^{\prime} \mathrm{W}$; Boulder City (Nevada), $35^{\circ} 59^{\prime} \mathrm{N}, 114^{\circ} 50^{\prime} \mathrm{W}$; Tucson (Arizona), $32^{\circ} 15^{\prime} \mathrm{N}, 110^{\circ} 50^{\prime} \mathrm{W}$.

Practically all the observations in table 1 were obtained by new readings from all available seismograms, and earlier lists, e.g., of Richter (1955, p. 196), were checked.

\section{Methods}

Plotting the observations for a given station on a map of the aftershock area, we find an apparently very irregular pattern with no possibility of separating areas giving compressions from those giving dilatations. Frequently, significantly opposite motions are obtained from the same epicenter. This is in sharp contrast to the usually regular distribution of initial motion obtained in studies of distant earthquake areas (see, e.g., Bath, 1952). A probable reason is that in the present study we are concerned with shocks of usually much lower magnitude than that for distant shocks. The weaker shocks may be expected, especially in an aftershock series, to occur on a large number of minor and auxiliary faults oriented in a variety of directions, whereas larger shocks are assumed to occur on the larger faults which conform better to the regional stress pattern. The complication from the fact that the first motion at nearer stations refers to the direct longitudinal wave (p), at more distant stations to the longitudinal wave refracted twice at the Mohorovičic boundary $(\mathrm{Pn})$, is insufficient as an explanation, because the irregularity mentioned is observed at all stations, including those with only Pn observations.

In view of the mechanical complexity of the aftershock area, it was necessary first to study each shock individually. This was done by plotting the observations for each shock separately, with the epicenter at the origin and station azimuth and distance as polar coördinates. A modified technique mainly for distant observations was recently developed by Bath (1958), and the ideas expressed there may easily be transferred to the present representation, which is better when only observations of near stations are used. In each case nodal lines were drawn, i.e., lines separating compressional areas from dilatational (or lines of zero amplitude for the $\mathrm{P}$ waves). It is obvious that the nodal lines are the intersections (FF and MM respectively) of the earth's surface with the fault plane and with a plane perpendicular to the direction of slip.

We use the following notation:

$a_{F}, a_{M}=$ the perpendicular distances from the epicenter E to FF and MM respectively

$\gamma_{F}, \gamma_{M}=$ the respective dips of the fault plane and of the plane perpendicular to slip

$\alpha \quad=$ the angle between FF and MM

$\beta \quad=$ the angle in the fault plane between dip and slip

$h \quad=$ the focal depth

The following relations hold, assuming rectilinear slip on a plane fault surface:

$$
\begin{gathered}
\tan \gamma_{F}=h / a_{F} ; \quad \tan \gamma_{M}=h / a_{M} \\
\cos \alpha=\cot \gamma_{F} \cdot \cot \gamma_{M} ; \quad \cos \beta=\cos \gamma_{M} / \sin \gamma_{F} \\
h=\left(a_{M} a_{F} / \cos \alpha\right)^{\frac{1}{2}}
\end{gathered}
$$


The different special cases are as follows:

1. Strike slip:
A. Vertical fault: $a_{F}=0 ; a_{M}=0 ; \alpha=90^{\circ}$.
B. Horizontal fault: $a_{F}=\infty ; a_{M}=0 ; \alpha$ indeterminate.
C. Inclined fault: $a_{F} \neq 0, \infty ; a_{M}=0 ; \alpha=90^{\circ}$.

2. Dip slip:

A. Vertical fault: $a_{F}=0 ; a_{M}=\infty ; \alpha$ indeterminate.

B. Horizontal fault: excluded.

C. Inclined fault: $a_{F} \neq 0, \infty ; a_{M} \neq 0, \infty ; \alpha=0^{\circ}$. The distance between FF and MM in this case is $a_{F}+a_{M}=2 h / \sin 2 \gamma_{F}$.

3. Combined slip:
A. Vertical fault: $a_{F}=0 ; a_{M} \neq 0, \infty ; \alpha=90^{\circ}$.
B. Horizontal fault: excluded.
C. Inclined fault: $a_{F^{F}} \neq 0, \infty ; a_{M} \neq 0, \infty ; \alpha \neq 0^{\circ}, 90^{\circ}$. This is the most general case.

Applying these considerations to our case, we find that for $h$ as low as $16 \mathrm{~km}$. the station net needs to be much closer in order to permit detection and determination of $a_{F}, a_{M}$. The nodal lines FF and MM can often be displaced parallel to themselves and still satisfy the observations as well as when they pass through E. Therefore it is impossible to make complete fault-plane solutions in the present case.

In our study we can only distinguish three types:

a. Uninodal type, i.e., $1 \mathrm{~B}, 2 \mathrm{~A}$, or $2 \mathrm{C}$ above, usually $2 \mathrm{C}$, whereas $1 \mathrm{~B}$ seems to be excluded in our study. In fact, $2 \mathrm{C}$ may be considered as a binodal parallel type.

b. Binodal perpendicular type, i.e., $1 \mathrm{~A}, 1 \mathrm{C}$, or $3 \mathrm{~A}$.

c. Binodal nonperpendicular type, i.e., $3 \mathrm{C}$.

In addition, we have to consider the complication from the fact that at the nearer stations the first longitudinal wave is $\mathrm{p}$, and at more distant stations Pn. This was pointed out by Wadati (1927) and extensively developed by Kawasumi (1934). With the notation

$$
\begin{aligned}
& H=\text { the crustal thickness down to the Mohorovičic boundary } \\
& v_{1}=\text { the longitudinal wave velocity in the crust } \\
& v_{2}=\text { the longitudinal wave velocity immediately below the crust } \\
& i=\text { the angle of incidence, defined by } \sin i=v_{1} / v_{2}
\end{aligned}
$$

we find the following expression for the critical distance $\Delta_{c}$, where $\mathrm{p}$ and $\mathrm{Pn}$ arrive simultaneously, neglecting the earth's curvature:

$$
\Delta_{c}=(2 H-h) \tan i\left[1+\left(1+\frac{4 H(H-h)}{(2 H-h)^{2} \tan ^{2} i}\right)^{\frac{1}{2}}\right]
$$

For distances $\Delta<\Delta_{c}$ the first wave is $\mathrm{p}$, and for $\Delta>\Delta_{c}$ it is Pn. It is to be noted that $\Delta_{c}$ is not necessarily the same in all directions from the epicenter, mainly owing to variations in $H$. Combining the $\Delta_{c}-$ formula with the earlier considerations, it is an easy matter to construct the theoretical distribution of initial motion under different circumstances.

In spite of the fact that the observations are insufficient to give complete solutions, they nevertheless usually define the directions of the nodal lines, one of which represents the fault trace, and they give the direction of the dominant motion. 
TABLE 2

Solutions

\begin{tabular}{|c|c|c|c|c|c|c|}
\hline No. & $\begin{array}{l}\text { Uni- } \\
\text { nodal }\end{array}$ & $\underset{\substack{\mathrm{Bi}-\\
\text { nodal }}}{\mathrm{B}}$ & $\Phi$ & Motion & Exceptions & Remarks \\
\hline & & & deg. & & & \\
\hline 40 & $x$ & $x$ & $\begin{array}{l}48 \\
38\end{array}$ & $\begin{array}{l}\text { sd } \\
1\end{array}$ & - & $\begin{array}{l}\text { Two solutions offered, the uninodal } \\
\text { possibly slightly better, consid- } \\
\text { ering Pasadena }\end{array}$ \\
\hline 46 & $x$ & & 66 & sd & -— & \\
\hline 58 & & $x$ & 65 & $\mathbf{r}$ & - & \\
\hline 59 & & $x$ & 51 & 1 & - - & Case 1C \\
\hline 60 & $x$ & & 51 & sd & —— & \\
\hline 61 & & $x$ & 57,132 & $\mathbf{r}$ & Fresno & Case $3 \mathrm{C}$ \\
\hline 62 & & $x$ & 44 & 1 & -— & \\
\hline 63 & & $x$ & 17 & 1 & -— & \\
\hline 64 & & $x$ & 58 & 1 & - - & \\
\hline 65 & & $x$ & $(50)$ & 1 & - — & \\
\hline 66 & & $x$ & 30 & 1 & $\ldots$ & \\
\hline 68 & & $x$ & 33 & 1 & $-\ldots$ & \\
\hline 69 & & $x$ & 10 & 1 & - - - & \\
\hline 70 & & $x$ & 46 & 1 & - - & \\
\hline 71 & $x$ & & 38 & sd & - & \\
\hline 73 & & $x$ & 67 & $\mathrm{r}$ & - - & Case 1C \\
\hline 74 & & $x$ & 53,113 & $\mathrm{r}$ & Mt. Hamilton & Case $3 \mathrm{C}$ \\
\hline 75 & $x$ & & 50 & sd & - & \\
\hline 76 & & & & & & Too few observations \\
\hline 77 & $x$ & & (43) & $\mathrm{su}$ & -— & $\begin{array}{l}\text { First motion could be } \mathrm{p} \text { at China } \\
\text { Lake }\end{array}$ \\
\hline 78 & $x$ & & 80 & su & -_- & Possibly case $2 \mathrm{C}$ \\
\hline 79 & & $x$ & (80) & $\mathrm{r}$ & Palomar & $\Phi$ could be as large as $95^{\circ}$ \\
\hline 80 & & $x$ & $(80)$ & $\mathbf{r}$ & Mt. Hamilton & $\Phi$ could be as large as $95^{\circ}$ \\
\hline 81 & & $x$ & $(84)$ & $\mathrm{r}$ & - & $\Phi$ could be as large as $98^{\circ}$ \\
\hline 82 & & $x$ & 44 & 1 & - - & \\
\hline 83 & $x$ & $x$ & $\begin{array}{c}80 \\
(80)\end{array}$ & $\begin{array}{l}\mathbf{r} \\
\text { su }\end{array}$ & Mt. Hamilton & $\begin{array}{l}\text { Two solutions offered } \\
\text { First motion could be } p \text { at Santa } \\
\text { Barbara in second case, or case } \\
2 \mathrm{C} \text { could give the solution }\end{array}$ \\
\hline 84 & $x$ & & $(80)$ & su & $-\ldots$ & \\
\hline 85 & & $x$ & 36 & 1 & $-\cdots$ & \\
\hline 86 & & $x$ & 43,145 & I & - - & Case 3C \\
\hline 87 & & $x$ & 32 & 1 & - - & \\
\hline 88 & & $x$ & 45 & 1 & 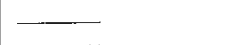 & \\
\hline 89 & $x$ & & $(80)$ & sd & Mt. Hamilton & \\
\hline 97 & & $x$ & 60 & $\mathrm{r}$ & $\begin{array}{l}\text { Mt. Hamilton, } \\
\text { Boulder City }\end{array}$ & $\begin{array}{l}\text { Some indication of a double motion } \\
\text { (possibly two shocks after each } \\
\text { other), which could also explain } \\
\text { the exceptions }\end{array}$ \\
\hline 113 & $x$ & & 18 & sd & - - & \\
\hline 115 & & $x$ & 59 & 1 & - _ & Possibly case $1 \mathrm{C}$ \\
\hline 116 & & $x$ & 14 & 1 & - - & \\
\hline 117 & & $x$ & 45 & 1 & Chuchupate & \\
\hline
\end{tabular}


TABLE 2-Continued

\begin{tabular}{|c|c|c|c|c|c|c|}
\hline No. & $\begin{array}{c}\text { Uni- } \\
\text { nodal }\end{array}$ & $\begin{array}{c}\text { Bi- } \\
\text { nodal }\end{array}$ & $\Phi$ & Motion & Exceptions & Remarks \\
\hline & & & deg. & & & \\
\hline 118 & & $x$ & 31,158 & 1 & - - & Case $3 \mathrm{C}$ \\
\hline 119 & & $x$ & 38 & 1 & $-\cdots$ & \\
\hline 120 & & $x$ & 60 & 1 & $--\overline{-1}$ & \\
\hline 140 & & $x$ & 43 & $\mathbf{r}$ & Chuchupate, Fresno & \\
\hline 141 & & $x$ & 53 & $\mathbf{r}$ & Barrett & Case $3 \mathrm{~A}$ \\
\hline 142 & & $x$ & 63,130 & $\mathbf{r}$ & -1 & $\begin{array}{l}\text { Case } 3 \mathrm{C} \text {; no reliable solution } \\
\text { possible }\end{array}$ \\
\hline 143 & & $x$ & $(83)$ & $\mathrm{r}$ & $-\cdots$ & Case $3 \mathrm{~A}$ \\
\hline 144 & $(x)$ & & $(70)$ & su & Santa Barbara & Solution uncertain \\
\hline 155 & & $x$ & 40 & 1 & $-\cdots$ & Case $1 \mathrm{C}$ \\
\hline 161 & & $x$ & 44 & 1 & Haiwee & $\begin{array}{l}\text { The exception could be eliminated, } \\
\text { assuming case } 3 \mathrm{C} \text {, giving } \Phi=16^{\circ} \\
\text { and } 134^{\circ}\end{array}$ \\
\hline 177 & & $x$ & $(80)$ & 1 & Havilah & \\
\hline 194 & & $x$ & 49 & $\mathrm{r}$ & $\begin{array}{l}\text { Palo Alto, Big Bear, } \\
\text { Chuchupate }\end{array}$ & \\
\hline 216 & & $x$ & 15 & $\mathrm{r}$ & $-\cdots$ & \\
\hline 241 & & $x$ & $(62)$ & 1 & - - & $\Phi$ could be as large as $102^{\circ}$ \\
\hline 266 & & $x$ & 46 & 1 & $-\ldots$ & \\
\hline 275 & & $x$ & 72 & 1 & - & \\
\hline 276 & & $x$ & 50 & 1 & - & \\
\hline 279 & & $x$ & 60 & 1 & Fort Tejon, Isabella & \\
\hline 281 & & $x$ & 35 & $\mathrm{r}$ & $-\ldots$ & \\
\hline 285 & $x$ & & 54 & $\mathrm{sd}$ & $\longrightarrow$ & \\
\hline
\end{tabular}

\section{Results}

The solutions are compiled in table 2. "Binodal" means perpendicular binodal, unless otherwise mentioned. The following notation is used:

$\Phi=$ the angle in the horizontal plane between the meridian and a nodal line, measured from $\mathrm{N}$ over $\mathrm{E}$; in the binodal perpendicular case only the smallest $\Phi$ is given; $(\Phi)$ indicates that $\Phi$ may be in error by more than $10^{\circ}$, otherwise $\Phi$ is accurate to within $5^{\circ}-10^{\circ}$

$\mathrm{r}=$ right-hand strike slip

$1=$ left-hand strike slip, both $\mathrm{r}$ and $\mathrm{l}$ referring to the direction $\Phi$

$\mathrm{su}=$ southeast side moves upward

$\mathrm{sd}=$ southeast side moves downward

Only significant exceptions are given in table 2 . They may be due:

i) to occurrence of $p$ instead of Pn for the nearest stations,

ii) to situation close to a nodal line,

iii) to unknown reversals of the connections of seismometer to galvanometer.

The nodal lines have been drawn so as to give a minimum number of exceptions.

The results are shown on a map, figure 1, where usually only those nodal lines have been chosen which are most nearly parallel to the main fault, assuming these nodal lines to represent FF. 
In contrast to the dominating dip slip in the main earthquake, strike slip predominates in the aftershocks. On the main White Wolf fault and to the south of it, left-hand strike slip dominates in conformity with the general motion pattern of the whole region (Benioff, 1955b, fig. 2). North of the main fault, on the other hand, the motion is almost exclusively right-hand strike slip or dip slip. We interpret this as a secondary or auxiliary release of strain, caused by the dominant left-hand

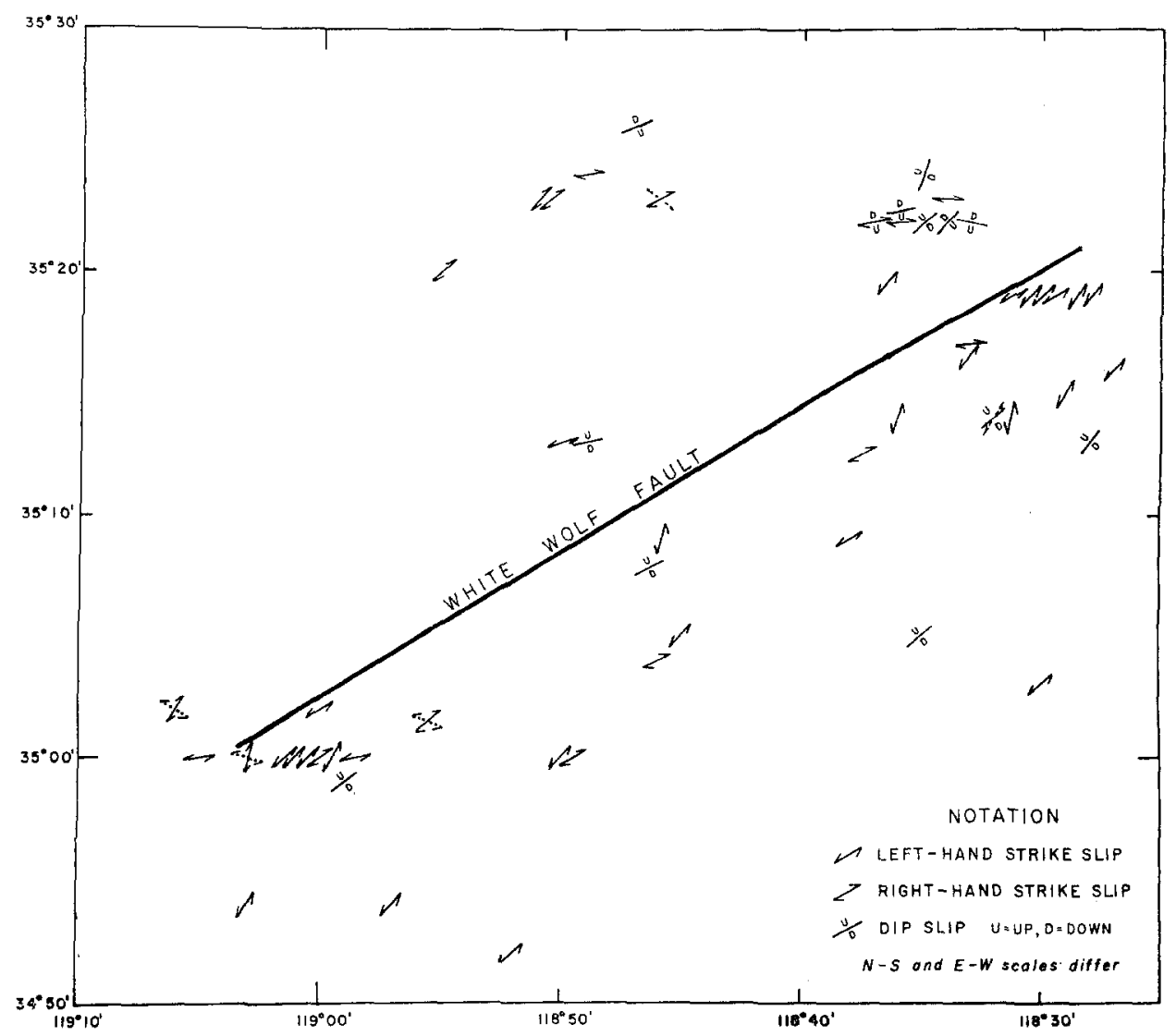

Fig. 1. Map of the aftershock area showing the individual solutions.

strike slip. The left-hand strike slip on the main fault will give rise to a strain pattern, which at some distance from the fault will be released as a right-hand strike slip. This secondary release could be expected on either side of the main fault; in the present case the secondary release occurred only on the northern side. In this connection it is of interest to note that there was a delay in the release on the northern side of thirty-seven hours after the great shock. After that time both mechanisms were in operation simultaneously, causing a change of the total strain release thirtyseven hours after the main earthquake (Benioff, 1955a). This occurrence was covered before and after by studying shocks nos. 58-89 inclusive. The second phase in the strain-release curve began with no. 75 , initiating the activity around $35^{\circ} 22^{\prime}$ $\mathrm{N}, 118^{\circ} 35^{\prime} \mathrm{W}$, with right-hand strike slip and dip slip. These shocks also exhibit a different appearance on the records. 
The two exceptions with left-hand strike slip on the northern side, i.e., nos. 155 and 275, are both located closer to the main White Wolf fault. No. 155 is also exceptional in originating at greater depth (around $23 \mathrm{~km}$.) than the majority of the aftershocks, which occurred around $16 \mathrm{~km}$. depth. No. 275 is of interest because, like no. 89 , it is situated within an area of considerably reduced activity immediately north of the main fault.

Aftershocks with dominating dip-slip motion are in the minority but occur in most parts of the aftershock area. It is of interest to note that, unlike what occurred at the time of the main shock, the southeastern side usually has a downward motion relative to the northwestern side. Exceptions occur only in the limited area around $35^{\circ} 22^{\prime} \mathrm{N}, 118^{\circ} 35^{\prime} \mathrm{W}$, initiated by no. 75 .

On July 25,1952 , a series of shocks occurred around $35^{\circ} 19^{\prime} \mathrm{N}, 118^{\circ} 30^{\prime} \mathrm{W}$, near the northeastern end of the active fault (nos. 115, 117-120) at an approximate depth of only $10 \mathrm{~km}$. These shocks exhibit exclusively a dominating left-hand strike slip, agreeing with the main motion in the region, and consistent with the suggestion that they represent a terminal extension of the original faulting.

A series of shocks on July 29, 1952, i.e., nos. $140-144$, near $35^{\circ} 23^{\prime} \mathrm{N}, 118^{\circ} 50^{\prime} \mathrm{W}$ are of special interest because, although belonging to a very limited area, they showed definitely opposite initial motions at some stations though not at others. It is clear from the map that these apparently contradictory cases fit very well into a coherent motion pattern with right-hand strike slip. The reversals of motion at several stations are attributable to somewhat different orientation of the faults in the different cases.

Several shocks were studied because of their location near the epicenter of the main earthquake at $35^{\circ} 00^{\prime} \mathrm{N}, 119^{\circ} 02^{\prime} \mathrm{W}$. This group includes nos. $61,64,66,70$, $76,177,216,241,266,276,281$, and 285 . With dominating left-hand strike slip it is obvious that they generally do not agree with the mechanism of the main shock. No. 216 and no. 281 have been given with both nodal lines indicated, since it is not immediately obvious which of them is to be interpreted as FF. No. 281 (February 7,1954 ) is of interest as representing a possible extended faulting northwestward from the southwestern end of the White Wolf fault, which took place late in the series, probably beginning on January 12, 1954.

Two examples of approximately opposite motions in almost the same place are offered by nos. 62 and 73 and by nos. 86 and 97, in each pair the left-hand strike-slip motion corresponding to that occurring in the earlier shock.

The Walker Pass earthquakes of 1946 (Chakrabarty and Richter, 1949) have possibly some mechanical relation to the Kern County series. The first motions of longitudinal waves for the large foreshock and the main earthquake indicate a binodal perpendicular solution with $\Phi=35^{\circ}$ and left-hand strike slip, thus agreeing with the main regional motion pattern. The orientation $\Phi=35^{\circ}$ of $\mathrm{FF}$ is of interest, since it nearly coincides with the trend of the aftershock area and other structural features (loc. cit., fig. 1), thus indicating the existence of a fault with this direction; this could be the major front fault of the Sierra Nevada, with moderately steep westward dip. 


\section{RefERENCES}

Båth, M.

1952. "Initial Motion of the First Longitudinal Earthquake Wave Recorded at Pasadena and Huancayo," Buill. Seism. Soc. Am., Vol. 42, No. 2, pp. 175-195.

1958. "Polar Graphs of Initial Motions at an Earthquake Source," Bull. Seism. Soc. Am., Vol. 48, No. 2, pp. 129-131.

Benioff, $\mathrm{H}$.

1955a. "Mechanism and Strain Characteristics of the White Wolf Fault as Indicated by the Aftershock Sequence," California Division of Mines, Bull. 171, pp. 199-202.

1955b. "Relation of the White Wolf Fault to the Regional Tectonic Pattern," ibid., pp. 203-204. Chakrabarty, S. K., and C. F. Richter

1949. "The Walker Pass Earthquakes and Structure of the Southern Sierra Nevada," Bull. Seism. Soc. Am., Vol. 39, No. 2, pp. 93-107.

Dehlinger, $\mathrm{P}$.

1952. "Shear-wave Vibrational Directions and Related Fault Movements in Southern California Earthquakes," Bull. Seism. Soc. Am., Vol. 42, No. 2, pp. 155-173.

Gutenberg, B.

1941. "Mechanism of Faulting in Southern California Indieated by Seismograms," Bull. Seism. Soc. Am., Vol. 31, No. 4, pp. 263-302.

1955a. "Seismograph Stations in California," California Division of Mines, Bull. 171, pp. 153156.

1955b. "The First Motion in Longitudinal and Transverse Waves of the Main Shock and the Direction of Slip," ibid., pp. 165-170.

Kawasumi, $\mathrm{H}$.

1934. "Amplitude of Seismic Waves with the Structure of the Earth's Crust and Mechanisms of Their Origin," Bull. Earthq. Res. Inst., Vol. 12, pp. 660-705.

Richter, C. F.

1955. "Foreshocks and Aftershocks," California Division of Mines, Bull. 171, pp. 177-197.

Wadati, $\mathrm{K}$.

1927. "On the Mohorovičić Wave Observed in Japan," Geophys. Mag., Vol. 1, pp. 89-96.

Seismologtcal Laboratory,

California Institute of Thchnology,

Pasadena, California

(Division of Geological Sciences, contribution no. 826.) 